\title{
Characterization of Lipid and Cell Membrane Organization by the Fluorescence Correlation Spectroscopy Diffusion Law
}

\author{
Xue Wen Ngac, Nirmalya Bag ${ }^{\mathrm{bc}}$, and Thorsten Wohland ${ }^{\star a b c}$
}

\begin{abstract}
The plasma membrane organization of live cells defines a plethora of cellular processes important for cell functionality. Many membrane structures that define this organization exist at a spatial resolution below the optical diffraction limit and are highly dynamic. Therefore, a method with millisecond time resolution and nanometer spatial resolution is required for the investigation of plasma membrane organization. However, spatial and temporal resolutions of the currently available biophysical techniques are often mutually exclusive. In a novel realization, Lenne and coworkers developed a spot-variation modality of fluorescence correlation spectroscopy (FCS), also known as FCS diffusion law, to harvest nanoscopic information from microscopic measurements. The FCS diffusion law, so far, has been instrumental to decode the physico-chemical origin of membrane organization and its relationship with biological processes. Overall, the structural information of plasma membrane obtained by FCS diffusion law provides a better understanding of its coupling to the underlying cellular processes.
\end{abstract}

Keywords: Fluorescence correlation spectroscopy · FCS diffusion law · Lipid raft model · Membrane dynamics - Membrane organization · Picket-fence model

\section{Introduction}

The cellular plasma membrane is a highly complex fluid structure with multiple levels of organization at different spatiotemporal scales. It consists of a lipid bilayer with proteins either adherent to the bilayer surface or spanning the membrane and which is coupled over some of these proteins to the cytoskeleton. Artificial lipid bilayers were demonstrated to have lateral fluidity by experiments in the 1960 s. ${ }^{[1]}$ The same lateral fluidity was then shown experimentally by Frye and Edidin (1970) for cell membranes. They virus-fused human and mouse cells, which were fluorescently labeled with different fluorophores, and observed the intermixing of membrane components between both cells as a result of diffusion of these membrane

\footnotetext{
${ }^{*}$ Correspondence: Dr. T. Wohland ${ }^{\text {abc }}$

Tel.: +65 65161248

E-mail: twohland@nus.edu.sg

aDepartment of Chemistry

bDepartment of Biological Sciences

cNUS Centre for Bio-Imaging Sciences

National University of Singapore

Blk S1A, Level 2, Lee Wee Kheng Building

14 Science Drive 4, Singapore 117557
}

components imparted by lateral fluidity of the membrane. ${ }^{[2]}$ The fluidity of the lipid matrix is influenced by lipid-lipid, lipid-protein and protein-protein interactions as well as cytoskeleton confinement. The first widely accepted model, the socalled 'fluid mosaic model', to describe plasma membrane organization was proposed by Singer and Nicolson in 1972. [3] It describes the plasma membrane as a two-dimensional homogeneous dynamic fluid mosaic structure with globular integral proteins randomly distributed at long range throughout a lipid bilayer (Fig. 1a). These integral proteins and lipids undergo lateral diffusion due to the fluidity of the plasma membrane. The rate of translational diffusion of integral proteins in the membrane depends mainly on the effective viscosity sensed by the proteins as a result of the arrangement of surrounding lipids to form the bilayer matrix. This means that long-range ordering of lipids and proteins does not exist in the plasma membrane and suggests that all membrane molecules are homogeneously distributed in the plasma membrane and undergo simple Brownian lateral diffusion.

However, further studies put the concept of a homogeneous plasma membrane into question. In 1982, Karnovsky et al. first described the presence of lipid domains in membranes where lipids undergo non-Brownian diffusion and are transiently confined in the domains. ${ }^{[4]}$ They performed a series of experiments which included fluorescence lifetime decay studies on a lipophilic probe, 1,6-diphenyl-1,3,5-hexatriene (DPH), preferential phase-partitioning studies of two groups of free fatty acids and membrane structure perturbation studies via fluorescence polarization of DPH with both groups of free fatty acids to probe the heterogeneity of both model and plasma membranes. In the fluorescence lifetime study of single phase dipalmityl lecithin (DPL) and dilauroyl lecithin (DLL) lipid vesicles, two decay components were detected, each corresponding to their respective single-phase decay components. On the other hand, a DPL and DLL mixed lipid phase resulted in a third decay component attributed to possible quenching of DPH at the boundary between domains due to binding of DPH with water molecules in the membrane. Multiple decay components were also found in plasma membranes of different cell lines, which supported the existence of site heterogeneity and therefore lipid domains in the plasma membrane. They also traced the direction of change in the melting temperatures of fluid and gel lipid phases caused by preferential phase-partitioning of two groups of fatty acids: $c i s$-unsaturated (fluid-phase preference) and trans-unsaturated or saturated (gel-phase preference) fatty acids, and monitored the effects of both groups of fatty acids on perturbing the structures of model and plasma membranes by fluo- 


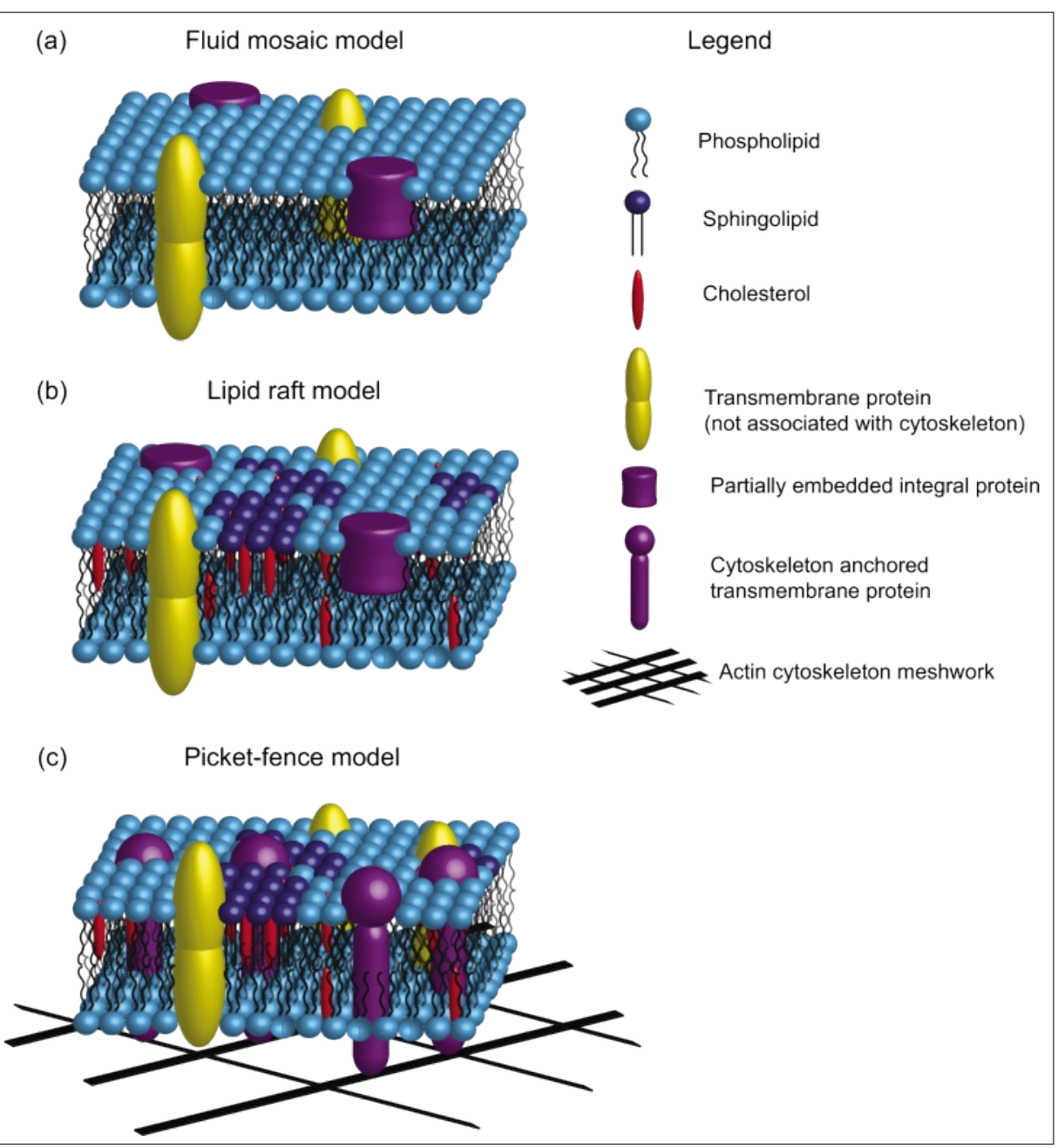

Fig. 1. Schematics of membrane organization models. (a) Fluid mosaic model with globular integral proteins (yellow and purple) distributed randomly throughout the lipid bilayer (light blue). (b) Lipid raft model where the lipid bilayer is laterally organized with sphingolipid (dark blue) and cholesterol (red) enriched small domains, called 'lipid rafts', phase-separated from the surrounding phospholipid matrix (light blue). (c) Picket-fence model with transmembrane proteins (purple and yellow) anchored and aligned respectively along the actin cytoskeleton (black meshwork) as rows of obstacles called 'pickets' while the actin cytoskeleton acts as 'fences'.

rescence polarization of DPH which also provided indirect evidence of a heterogeneous lateral distribution of lipids in the form of lipid domains in model and plasma membranes.

In parallel to the foregoing biophysical methods, early biochemical studies also revealed the presence of membrane domains consisting of nonionic detergent (Triton-X-100) resistant membrane (DRM) constituents enriched in cholesterol and sphingolipids. ${ }^{[5-8]}$ This motivated the concept of 'rafts' in cell membranes which was eventually proposed by Simons and Ikonen in 1997.[9] The lipid raft model describes the plasma membrane as a highly dynamic and heterogeneous fluid bilayer. This fluid bilayer is laterally organized with small domains called 'lipid rafts' that are enriched in sphingolipid and cholesterol and are phase-separated from the surrounding fluid lipid matrix occupied mainly by unsaturated phosphatidylcholine molecules as shown in Fig. 1b. The presence of such lipid rafts led to the non-Brownian diffusion behavior of membrane components including lipids and proteins in the plasma membrane. Throughout the years, many studies suggested functions of lipid rafts in cellular processes ranging from membrane trafficking, endocytosis, signal transduction to host-pathogen interactions. ${ }^{[10-13]}$ However, the definition and functions of lipid rafts remain controversial to date. A general consensus was proposed in the 'Keystone Symposium on Lipid Rafts and Cell Function, 2006' where the term 'lipid raft' was recoined as 'membrane raft' and defined as "Membrane rafts are small 10$200 \mathrm{~nm}$, heterogeneous, highly dynamic, sterol- and sphingolipid-enriched domains that compartmentalize cellular processes. Small rafts can sometimes be stabilized to form larger platforms through protein-protein and protein-lipid interactions".[14]

At the same time, it was not understood why lipids in the plasma membrane possess a diffusion rate of 5-100 times smaller than that in model membranes that could not be explained simply by protein crowding effect. ${ }^{[15-17]}$ Upon better understanding of the plasma membrane organization from single particle tracking (SPT) and single fluorophore video imaging experiments conducted at high time resolution, Fujiwara et al. addressed the above-mentioned issue in 2002 by establishing the 'picket-fence model' . ${ }^{[18]}$ The picket-fence model regards transmembrane proteins which are anchored and aligned along the membrane cytoskeleton as rows of obstacles (pickets) that sterically hinder the free diffusion of membrane components. The anchored transmembrane proteins are immobilized by the membrane cytoskeleton, which increases the local membrane viscosity due to hydrodynamic friction effects. The hydrodynamic friction slows down the diffusion of membrane components around the immobilized transmembrane proteins. On the other hand, the cytoplasmic surface of the plasma membrane is spatially segmented into cholesterol-independent compartments created by its interaction with actin cytoskeleton fences (Fig. 1c). Partial actin depolymerization was conducted by latrunculin-A treatment in which the compartment area was observed to increase by a factor of $\sim 2$, thereby confirming the significant role played by the actin cytoskeleton in the formation of fences. In the picket-fence model, the plasma membrane molecules undergo short-range free diffusion within a fence similar to free diffusion in model membranes. They also undergo long-range hop diffusion across multiple fences which is much slower due to the separation of fences by physical barriers as well as the presence of diffusion barriers provided by the cytoskeleton-bound transmembrane proteins. These factors explain the retardation of diffusion of plasma membrane lipids by a factor of 5-100 compared with model membranes.

Both models stated above hypothesize that membrane components exhibit non-Brownian diffusion behavior either by transient confinement into membrane rafts or by compartmentalization inside the cytoskeleton meshwork. To investigate the relevant spatial and temporal scales, a method with millisecond time resolution and nanometer spatial resolution is required. Unfortunately, most of the existing techniques can achieve either good spatial or good time resolution but not both simultaneously. In this context, the FCS diffusion law is a welcome entry in the arena of fluorescence-based methods. In the current review, we first provide a brief overview on the currently available fluorescence-based methods used to study membrane organization. Subsequently, we describe the detailed theory of the FCS diffusion law and its different technical variations. Finally, 
we discuss a broad spectrum of applications of the FCS diffusion law in model and live cell membranes.

\section{Fluorescence Detection Methods to Probe Membrane Organization}

Although a range of non-optical techniques have been used to characterize membrane organization such as electron spin resonance (ESR) spectroscopy, ${ }^{[19-21]}$ electron microscopy (EM), ${ }^{22,23]}$ nuclear magnetic resonance (NMR) ${ }^{[24-27]}$ and others, ${ }^{[28]}$ we will focus on optical fluorescence fluctuation techniques here, which have been at the forefront recently in the elucidation of membrane structure and dynamics.

Due to the caveats associated with nonoptical techniques such as poor temporal resolution, invasive sample preparation and measurement steps which introduce artifacts and stringent requirements in sample preparation, less invasive optical techniques were explored instead. In particular, optical techniques utilizing fluorescence as their mode of detection have emerged as promising avenues to study membrane organization due to the numerous advantages they possess. ${ }^{[29]}$ Fluorescence techniques are highly sensitive and selective owing to the possibility of labeling specific intracellular sites of interest with labels such as genetically engineered fluorescent proteins and fluorescent antibody-conjugated probes which target precise locations of biological samples. Furthermore, fluorescence techniques have a wide selection of well-established extrinsic (organic dyes and quantum dots) and intrinsic (fluorescent proteins) fluorescent labels which are easily applied to model and live biological samples. ${ }^{[30]}$ This provides a sound alternative to non-optical techniques for the study of membrane organization in model and cell membranes.

However, conventional fluorescence microscopy is diffraction-limited $(\sim 200$ nm spatial resolution) with limited time resolution, which neither allows direct visualization of cell membrane domains and compartments nor the observation of their dynamics. But several fluorescence spectroscopy techniques have very high temporal resolution, which provides at least indirect access to the organizational features of the membrane via their dynamics. Fluorescence recovery after photobleaching (FRAP) is one such fluorescence spectroscopy technique that has potentials showing the presence of micrometer-scale domains in model ${ }^{[31]}$ and live cell ${ }^{[32,33]}$ membranes. FRAP takes advantage of photobleaching to monitor the dynamics of fluorescently labeled molecules by tracking the fluorescence recovery of a photobleached region of interest in time and plotting a recovery curve. ${ }^{[34]}$ By fitting the recovery curves with appropriate models, dynamical information in terms of diffusion and interactions as well as the fraction of mobile and immobile fractions can be determined. FRAP measurements were done at varying spot sizes by changing the illumination beam radius and based on the dependence of the mobile fraction and apparent diffusion coefficient of the diffusing species on the spot size, the presence of membrane domains and their average size could be predicted. However, the predicted domain radii of human skin fibroblast cell membrane $(0.74 \pm 0.20 \mu \mathrm{m}$ for lipids and $0.25 \pm 0.10 \mu \mathrm{m}$ for proteins) and mouse hepatoma cell membrane $(0.41$ $\pm 0.20 \mu \mathrm{m}$ for lipids and $0.25 \pm 0.10 \mu \mathrm{m}$ for proteins) were well above the diffractionlimit which poses the question as to why direct fluorescence imaging did not show any domains in live cell membranes. This could possibly be due to the highly dynamic and transient formation of the membrane domains which did not allow them to be imaged directly. Nevertheless, FRAP suffers from several limitations such as the usage of high laser power for the photobleaching process which inevitably leads to photodamage of the biological sample and the prerequisite of a high amount of fluorophore which may not reflect physiological concentrations of biomolecules in the sample.[35]

The introduction of super-resolution microscopy extended the available spatial resolution down to $\sim 20-40 \mathrm{~nm}$. In spite of the improved spatial resolution of superresolution microscopy, there is unfortunately no report of any direct visualization of such membrane domains and compartments. Therefore, advanced optical spectroscopy techniques, which measure with low illumination laser powers at or close to physiological conditions, are still the best available tools to investigate the presence of membrane domains and compartments in the plasma membrane.

Single particle tracking (SPT) is an optical spectroscopy technique with sufficient temporal and spatial resolution which successfully measured membrane organization in the form of membrane domains and compartmentalization by the cytoskeleton. SPT analysis plots the time-averaged mean squared displacements (MSD) of several trajectories of a given probe against time and fits the plot with different diffusion models including free diffusion, confined diffusion and compartmentalized diffusion. The fitted plots were then classified into their respective diffusion states and characterized in terms of confinement area for confined diffusion in membrane domains and compartment size for compartmentalized diffusion due to interaction with the actin cytoskeleton. ${ }^{[36]}$ Recently, Bosch et al. implemented Bayesian inference on SPT data to distinguish different diffusion states. ${ }^{[37]}$ SPT is particularly useful in detecting membrane compartment sizes as shown by Murase et al. in multiple cell lines ranging from 30-230 nm via hop diffusion of colloidal-gold probed DOPE on the cell membranes of each cell line.[38] Membrane domain sizes of $0.7 \pm 0.02 \mu \mathrm{m}$ could also be measured in the human coronary artery smooth muscle (HASM) cell membrane by SPT. ${ }^{[39]}$ However, fast temporal resolution $(\sim 25 \mu \mathrm{s})$ is required to capture hop diffusion trajectories, which necessitates the use of colloidal gold labels to obtain sufficient signal-to-noise ratio during SPT. Large gold colloidal labels $(40 \mathrm{~nm})$ however can exhibit crosslinking effects on target molecules which could drastically affect particle movement of the probe and mask the underlying dynamics. ${ }^{[40-42]}$ Gold probes could also have interactions with the extracellular matrix, extracellular parts of some membrane proteins and lipid molecules in the outer leaflet of the plasma membrane, resulting in an inaccurate determination of membrane dynamics and organization. $[15,42,43]$

These limitations are partially solved by performing fluorescence correlation spectroscopy (FCS) measurements at multiple spatial scales to investigate submicron plasma membrane organization utilizing common fluorescent probes much smaller in size than gold probes. ${ }^{[4]}$ This method is known as the FCS diffusion law analysis.

\section{Theory of Fluorescence Correlation Spectroscopy (FCS) Diffusion Law}

FCS is a single molecule sensitive technique that statistically analyzes temporal fluctuations of fluorescence signals recorded within a small observation volume. ${ }^{[45-47]}$ An autocorrelation function (ACF) of the fluorescence fluctuations is then calculated, which contains information of the molecular processes such as diffusion of particles through the observation volume, chemical reactions, photophysical processes, or any process altering the fluorescence of the molecules involved. Upon fitting the experimental ACF curve with an appropriate theoretical model which takes account of the underlying processes, its dynamics can be quantified. FCS is widely used to determine molecular dynamics in the life sciences and in particular on membrane dynamics and was recently reviewed. ${ }^{48-50]}$ However, FCS also suffers from a range of disadvantages. Model selection in general requires some prior knowledge about the processes involved, which can lead to ambiguities, if several 
competing models provide similar fits. For example, it was shown that residuals obtained from the fitting of green fluorescent protein-linked glycosylphosphatidylinositol (GPI)-anchored protein (GFP-GPI) and fluorescently conjugated lipid probe BODIPY- $\mathrm{C}_{12}$-sphingomyelin (BODIPY$\mathrm{C}_{12}-\mathrm{SM}$ ) diffusion on plasma membranes with a two-component free diffusion model or a one-component anomalous diffusion model do not give a clear distinction between the models. ${ }^{[51]}$ The introduction of Bayes model selection to FCS facilitated model selection rendering it more robust and less subjective. ${ }^{[52-54]}$ In addition, conventional FCS is limited by the optical diffraction limit and thus could not provide information on spatial scales below about $200 \mathrm{~nm}$. However, as initially discussed, membrane domains are supposed to exist in size ranges well below that size.

This motivated Wawrezinieck et al. to propose an extension of FCS, called the FCS diffusion law, by conducting FCS measurements at variable spatial scales, [44] an approach that had been utilized earlier in FRAP measurements for detecting the presence of micrometer-scale domains in membranes. ${ }^{[32,33]}$ The FCS diffusion law leverages on the spatial dependence of the diffusion coefficient of membrane probes due to membrane structural features. In a freely diffusive system, the diffusion coefficient is independent of the spatial scale over which it is measured. In other words, the time molecules need to diffuse through an observation area is directly proportional to that area. However, in a heterogeneous system where diffusion is hindered by trapping of molecules or diffusive barriers, this relation does not hold anymore. Although the diffusion time is still linearly dependent on the observation area for large areas, it does not pass through the origin anymore (see Fig. 2). From the sign and the magnitude of the $y$-intercept, one can then deduce the nature of the diffusive process.

The FCS diffusion law was first demonstrated by simulations and proof-ofprinciple experiments which closely match with the current concept of plasma membrane organization (see Introduction section). FCS diffusion law analysis plots the apparent diffusion time $\left(\tau_{D}^{a p p}\right)$ of a probe obtained from the full width at half maximum (FWHM) of the ACFs obtained from FCS measurements against the illumination spot size $\left(\omega^{2}\right) \cdot \tau_{D}^{a p p}$ of a probe on a heterogeneous membrane consists of the convolution of all diffusion modes on the membrane such as hop diffusion, confined and free diffusion. The plot of $\tau_{D}^{a p p}$ versus $\omega^{2}$ of the simulations clearly defined three main scenarios of membrane dynamics dictated by existing membrane organization (Fig. 2).

i) For free diffusion: $\tau_{D}^{a p p}$ scales lin-

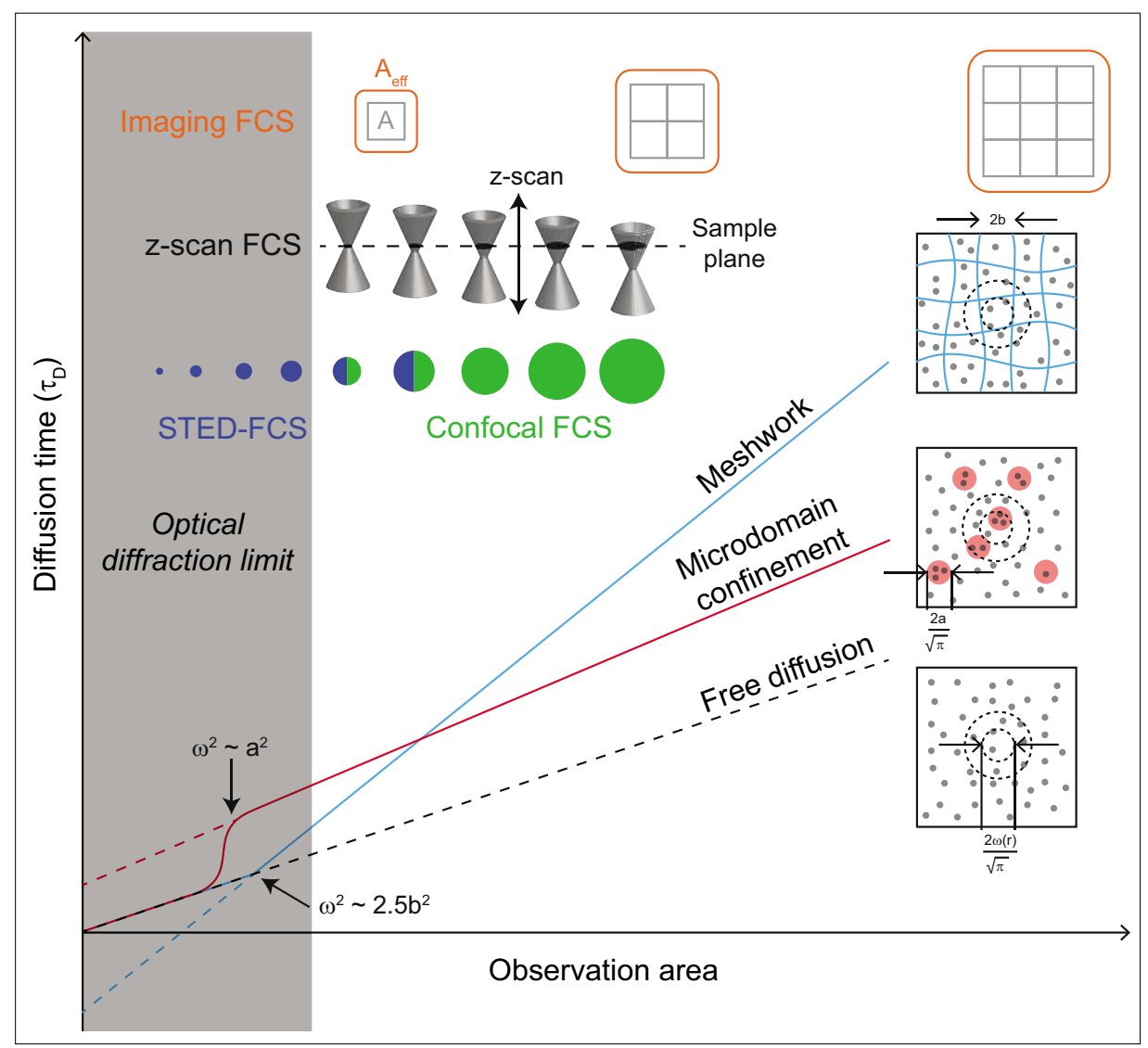

Fig. 2. FCS diffusion law plots for various diffusion modes in the membrane: free diffusion (black dotted line), hindered diffusion in microdomain confinement (red line) and hop diffusion in meshwork compartmentalization (light blue line). Range of spatial resolutions of the observation areas (in scale) for each experimental variation: STED-FCS (dark blue circles), confocal FCS (green circles), z-scan FCS and Imaging FCS (orange squares) are shown.

early with $\omega^{2}$ and passes through the origin (Fig. 2, black dotted line).

ii) For hindered diffusion with microdomain confinement: Assuming a circular domain size of $a^{2}$, at $\omega^{2} \leq 0.1 a^{2}, \tau_{D}^{a p p}$ scales linearly with $\omega^{2}$ and intersects the origin, indicating free diffusion. At $\omega^{2} \approx$ $a^{2}$, a transition region is observed where $\tau_{D}^{a p p}$ shows a non-linear relationship with $\omega^{2}$ due to complex diffusion processes as a result of barrier effects. Lastly at $\omega^{2} \geq$ $10 a^{2}, \tau_{D}^{a p p}$ scales linearly with $\omega^{2}$ again but with a higher slope and a strictly positive intercept. All these regimes are depicted in Fig. 2 as a red solid line. The values of the slope and intercept are influenced by several factors. One such factor is the confinement strength of the domain defined as $\tau_{\text {conf }} / \tau_{D}^{\text {domain }}$ where $\tau_{\text {conf }}$ is the average time needed for a probe placed in the middle of the domain to escape from it and $\tau_{D}^{\text {domain }}$ is the free diffusion time in a single domain. The confinement strength indicates the height of the energy barrier of a domain as a function of the probability of crossing the barrier and the size of the domain. Another factor affecting the slope and intercept is the density of microdomains (total area occupied by domains/ measurement area). The values of the slope and intercept are increasing functions of the density of microdomains, probability of the probe exiting the domains and the confinement strength while decreasing functions of the probability of the probe entering the domains.

iii) For hop diffusion due to meshwork compartmentalization: Assuming square meshes of length $2 b$, at $\omega^{2}<2.5 b^{2}, \tau_{D}^{a p p}$ scales linearly with $\omega^{2}$ and intersects the origin, indicating free diffusion. On the other hand, when $\omega^{2} \approx 2.5 b^{2}$, a short transition region is observed due to the physical barriers at the boundary of the mesh encountered by the probe which continues at $\omega^{2}>2.5 b^{2}$ where $\tau_{D}^{a p p}$ scales linearly with $\omega^{2}$ but with a higher slope and a strictly negative intercept. All these regimes are depicted in Fig. 2 as a light blue solid line. The absolute magnitudes of the intercept and the slope are increasing functions of the diffusion barrier height at the boundary of the mesh. In addition, the crossover point at $\omega^{2} \approx 2.5 b^{2}$ where the diffusion process switches from free to obstructed diffusion can be used to calculate the mesh size. The theory is described by Wawrezinieck et al. ${ }^{[44]}$

Since the diffraction-limited spot size created by conventional optical setups 
is significantly larger than either the microdomains or mesh sizes expected in the biological samples, transitions which lead to non-linearity in the FCS diffusion law plots for scenarios ii) and iii) cannot be observed directly. As shown in Fig. 2 (dotted lines), this can be semi-quantitatively resolved by extrapolating experimental FCS diffusion law plots to zero observation area $\left(\omega^{2}=0\right)$ to obtain the characteristic phenomenological intercept values $\left(\tau_{0}\right)$ for hindered diffusion with microdomain confinement ( $\tau_{0}>0$ ), hop diffusion due to meshwork compartmentalization $\left(\tau_{0}<0\right)$ and free diffusion $\left(\tau_{0}=0\right)$. The extrapolated FCS diffusion law plot is then given by

$$
\tau_{D}^{a p p}\left(\omega^{2}\right)=\tau_{0}+\frac{\omega^{2}}{4 D_{e f f}}
$$

where $D_{\text {eff }}$ is the effective diffusion coefficient defined as the inverse of the slope of the FCS diffusion law plot. $D_{\text {eff }}$ depends on the domain confinement strength, probe partitioning, density of domains and barrier height. Soon after its development, the analytical solution of the FCS diffusion law for meshworks was provided by Destainville where the hindered two-dimensional diffusion of a particle is simulated on a meshgrid of semi-permeable barriers which corresponds well with the scenario of hop diffusion due to meshwork compartmentalization. [55]

\section{Variations of FCS Diffusion Law}

Since its inception, the FCS diffusion law has been adapted to different types of instrumental setups for the specific type of investigations. We will describe the major implementations in the subsequent paragraphs briefly.

\subsection{Confocal FCS}

The most conventional way of performing FCS diffusion law analysis is through FCS measurements with a standard confocal microscope setup modified as follows. In order to create varying sizes of observation areas (Fig. 2, green circles), a diaphragm is placed between the laser beam expansion path and the dichroic mirror. This enables the underfilling of the microscope objective back aperture which decreases its apparent numerical aperture (NA). ${ }^{[4]} \mathrm{NA}$ is inversely proportional to the point spread function (PSF) of the objective, thus varying NA varies spot size. The same is also achieved by employing a motorized variable beam expander in the laser beam path which acts similarly to underfill the microscope objective by changing the collimated laser beam size. ${ }^{[56]}$ Multiple FCS measurements are then conducted sequentially at variable observation areas to plot the FCS diffusion law to understand membrane organization from the intercept values. As required for standard confocal FCS experiments, one should perform calibration measurements using standard calibration dyes to obtain the correct spatial dimension of each of the observation volumes prior to sample measurement.

\section{2 z-scan FCS}

Humpolíčková et al.[57] implemented the FCS diffusion law in an alternative FCS module, namely z-scan FCS, ${ }^{[58]}$ which still utilizes a standard confocal microscope. $\mathrm{z}$ scan FCS generates "variable measurement areas' by scanning and focusing along the $\mathrm{z}$-direction of the divergent laser beam to make multiple FCS measurements at different laser beam diameters (Fig. 2, grey laser beam waist). Essentially, this realization of FCS diffusion law does not need any additional optical element to create multiple observation sizes. In z-scan FCS, both the number of particles $(N)$ and diffusion time $\left(\tau_{\mathrm{D}}\right)$ of the probes in the illuminated membrane surface within the confocal volume have a parabolic dependence on the z-position of the membrane. By assuming a Gaussian beam profile, the $\mathrm{z}$-dependence of both $N$ and $\tau_{\mathrm{D}}$ is given by:

$$
N\left(\Delta z^{2}\right)=N_{0}\left(1+\frac{\lambda_{0}^{2} \Delta z^{2}}{\pi^{2} n^{2} \omega_{0}^{4}}\right)
$$

$$
\tau_{D}\left(\Delta z^{2}\right)=\frac{\omega_{0}^{2}}{4 D}\left(1+\frac{\lambda_{0}^{2} \Delta z^{2}}{\pi^{2} n^{2} \omega_{0}^{4}}\right)
$$

where $\lambda_{0}$ is the excitation light wavelength, $\omega_{0}$ is the beam waist radius of the excitation laser beam, $\Delta z$ is the distance between the z-positions of the sample and the laser beam waist, $n$ is the refractive index of the medium, $N_{0}=\pi C \omega_{0}^{2}$, where $C$ is the average surface concentration of the fluorescent probe in the illuminated membrane area, and $D$ is the absolute diffusion coefficient of the system under investigation. With respect to the original FCS diffusion law Eqn. (1) and by varying $\Delta z$ instead of spot size, Eqn. (3) gives the z-scan FCS diffusion law equivalence of Eqn. (1). Ganguly et al. plotted $\tau_{D}$ against $\Delta z^{2}$ according to Eqn. (3). ${ }^{59]}$ They first determined a $\Delta z^{2}$ value where $\tau_{D}=0$ for a freely diffusing sample. Diffusion time at this particular $\Delta z^{2}$ obtained after extrapolation of experimental $\tau_{D}$ against $\Delta z^{2}$ plot provides the FCS diffusion law intercept $\left(\tau_{0}\right)$

The Hof group combined Eqns (2) and (3) to derive the following Eqn. (4). In this case, one plots $\tau_{\mathrm{D}}$ against $N / N_{0}$ instead, ruling out the necessity of additional ex- periments on freely diffusing samples to determine $\tau_{0}$.

$$
\tau_{D}\left(\Delta z^{2}\right)=\tau_{0}+\frac{\omega_{0}^{2}}{4 D_{e f f}} \frac{N\left(\Delta z^{2}\right)}{N_{0}}
$$

\subsection{Imaging FCS}

Our group has recently implemented the FCS diffusion law in a camerabased platform of FCS called 'Imaging FCS' which was recently reviewed.[60,61] Imaging FCS combines a fast EMCCD or sCMOS camera with either total internal reflection (TIR) ${ }^{[62]}$ illumination or single plane illumination microscopy $(\mathrm{SPIM})^{[63]}$ schemes to achieve real-time spatial multiplexing of FCS measurements with moderate to high temporal resolution. This camera-based FCS modality takes advantage of the ability to perform binning of pixels post-acquisition to create multiple observation areas from a single FCS measurement. Imaging FCS thus eliminates the need to make multiple measurements at different observation areas which reduces both photophysical damage to the fluorescent probe by laser light irradiation and photo-induced detrimental effects to live samples. Furthermore, no additional optical instrument is required for varying the observation area.

The effective observation area $\left(A_{\text {eff }}\right)$ at different bin sizes in Imaging FCS can be accurately determined by convoluting the detection area $(A)$ of a given bin size (e.g. $1 \times 1,2 \times 2,3 \times 3$, etc. $)$, which is the area of the binned camera pixels, with the PSF of the optical system (Fig. 2, orange squares $\left(A_{\text {eff }}\right)$ and grey squares $\left.(A)\right)$. We have earlier established a robust method of determining the PSF of a microscope, ${ }^{[64]}$ taking advantage of the diffusion coefficient of homogeneous systems as an inherent property independent of PSF and measurement areas. As such, we modify the original confocal FCS diffusion law Eqn. (1) slightly into the Imaging FCS diffusion law equation as shown.

$$
\tau_{D}\left(A_{e f f}\right)=\tau_{0}+\frac{A_{e f f}}{4 D_{e f f}}
$$

Similarly, the intercept $\tau_{0}$ indicates the type of membrane organization present where a positive, negative and zero $\tau_{0}$ reflects hindered diffusion in microdomains, hop diffusion in meshwork compartmentalization and free diffusion respectively.

The spatial counterpart of Imaging FCS diffusion law was recently introduced by Di Rienzo et al., who used spatiotemporal image correlation spectroscopy (STICS) to deduce imaging MSD (iMSD) vs. timedelay plots. ${ }^{[65]}$ 


\subsection{STED-FCS}

FCS diffusion law was also realized in stimulated emission depletion (STED) fluorescence nanoscopy by Eggeling et al.[66] Spot size variation is achieved in STEDFCS by changing the STED beam intensity to get various observation area radii ranging between $15-240 \mathrm{~nm}$, which is well below the diffraction-limit.[67] STED-FCS diffusion law is so far the most advanced method that can provide direct evidence of domain sizes $(<20 \mathrm{~nm})$ and confinement time within a domain as a result of the roughly 5-10 times improved spatial resolution compared to diffraction-limited systems. By performing multiple STEDFCS measurements at varying spot sizes, the transition regimes which lead to nonlinearity in the FCS diffusion law plots for transiently confined diffusion due to domains and hop diffusion in meshwork compartmentalization can in principle be observed. Therefore, the STED-FCS diffusion law could essentially provide the most accurate representation of membrane organization in model and live cell membranes.

However, all of the above mentioned techniques suffer from their respective limitations. Firstly, the determination of the observation volume for confocal FCS measurement is calibration-dependent and thus cumbersome. FCS diffusion law plot by confocal FCS requires multiple sequential FCS measurements at different spot sizes which therefore do not provide real-time information of the membrane organization probed but a time-averaged interpretation of it. Moreover, multiple sequential measurements will subject the sample to undesired photophysical damage. Although being a calibration-free technique, $\mathrm{z}$-scan FCS suffers from the same disadvantage as confocal FCS in terms of time-averaging and damage of sample due to multiple sequential measurements conducted on the sample. On top of being a calibration-free technique, Imaging FCS rivals both confocal and z-scan FCS with respect to its ability to conduct pixel-binning post acquisition to obtain real-time information simultaneously with variable observation areas from a single FCS measurement. However, Imaging FCS suffers from having the poorest spatial resolution as compared to the rest of the techniques and offers limited options of spot sizes. Lastly, STED-FCS provides the best temporal and spatial resolution among all the techniques. Despite this advantage, STED-FCS is technically more demanding and is restricted to the use of specific membrane markers due to the high STED laser power required. STEDFCS also suffers from the disadvantages of multiple sequential measurements similar to confocal and z-scan FCS.

\section{Applications of the FCS Diffusion Law in Model and Cell Membranes}

The FCS diffusion law is widely applied in both model and cell membranes to characterize submicron membrane organization. We discuss these applications in the context of model and live cell membranes.

\subsection{Model Membranes}

Early applications of the FCS diffusion law on model membranes began with z-scan FCS by the Hof group using a DOPC:DOPS supported lipid bilayer (SLB) as proof-of-principle for $\mathrm{z}$-scan FCS diffusion law and a control for a free diffusive system. ${ }^{[58]}$ Subsequently, the FCS diffusion law found a wide range of applications with various instrumental setups to investigate organizational features of the membrane.

Heinemann et al. recently used the confocal FCS diffusion law to demonstrate the importance of the actin cytoskeleton to dictate membrane organization in a synthetic minimal model membrane. ${ }^{[68]}$ They determined and compared the diffusion modes for labeled lipid Atto647N-1,2-dioleoylsn-glycero-3-phosphoethanolamine (DOPE) and labeled protein cholera toxin B (CTxB)-Alexa647 probes for freestanding lipid membranes both in the absence and presence of actin. Free-standing membranes were created on holey silicon nitride EM supports via electrophoretic attraction of vesicles. ${ }^{[69]}$ In the absence of actin, it was found that the diffusion law plots for both lipid ( $\left.\tau_{0}=6 \pm 209 \mu \mathrm{s}\right)$ and protein $\left(\tau_{0}=62 \pm 329 \mu \mathrm{s}\right)$ probes intersect at the origin upon extrapolation to zero spot size, which corresponds to free diffusion as expected for free-standing membranes. On the contrary in the presence of actin, negative $\tau_{0}$ values for lipid $\left(\tau_{0}=-501 \pm\right.$ $241 \mu \mathrm{s})$ and protein $\left(\tau_{0}=-7880 \pm 2400\right.$ $\mu \mathrm{s})$ are obtained. This indicates a hindered diffusion with a transition from fast free diffusion to slow hindered diffusion due to meshwork compartmentalization for both lipid and protein probes by an actin mesh created on the free-standing membranes. In addition, the actin mesh seems to have stronger confinement effects for the protein than the lipid since the $\tau_{0}$ intercept value of the protein is more negative than that of the lipid. In another work by Favard et al., the FCS diffusion law was obtained both experimentally in a standard confocal setup and by Monte Carlo simulations to determine the membrane organization of single-phase and two-phase lipid multilamellar vesicles (MLVs), DMPC and DMPC/DSPC (8:2) respectively. ${ }^{[70]}$ From the experimental FCS diffusion law plots, negative $\tau_{0}$ values were obtained for both DMPC and DMPC/DSPC lipid membranes in their gel phases, indicating the presence of gel domains which behave like a meshwork. They also showed the temperature dependence of the formation and size of gel domains before and after their phase transitions. In the case of the twophase co-existing DMPC MLV, domains of two different spatial scales were revealed by experimental FCS diffusion law plots with mean domain of radii $50-80 \mathrm{~nm}$ and Monte Carlo simulated FCS diffusion law plots with mean domain radii of $2.5-5 \mathrm{~nm}$.

In the z-scan mode, FCS diffusion law was applied to show the selective formation of microdomains in anionic DOPC:DOPS (4:1) SLB upon addition of the positively-charged antimicrobial peptide, arenicin-1. ${ }^{[71]}$ Formation of microdomains was not observed in zwitterionic DOPC SLB where the lipids remain freely diffusing before and after addition of arenicin-1. The authors suggested that the formation of such microdomains could be the result of crosslinking between the anionic lipids and dynamic extended structures of the positively-charged arenicin-1. The importance of the formation of domains due to phase separation in model membranes by crosslinking of raft gangliosides GM1 and CTxB was also shown by the same group using z-scan FCS diffusion law. ${ }^{[72]}$ In another application of the z-scan FCS diffusion law, the temperature dependence of membrane organization of both the inner and outer leaflet of a DMPC membrane on a chitosan support at its phase transitions was characterized. ${ }^{[73]}$ At temperatures less than the phase transition temperature of DMPC $\left(\sim 27{ }^{\circ} \mathrm{C}\right)$, FCS diffusion law plots indicate hindered diffusion by microdomains which are likely gel phase-separated domains in both the inner and outer leaflets. At temperatures of $27{ }^{\circ} \mathrm{C}$ and above, both leaflets exhibit free diffusion behaviors at the fluid phase of DMPC, indicating the possible destruction of the gel phaseseparated domains.

In an attempt to increase spatial resolution below the diffraction-limit, nanometric apertures were used on a DOPC multilayer membrane which demonstrates free diffusion of the DOPC lipids from the FCS diffusion law plot. ${ }^{[74]}$ The application of FCS diffusion law below the diffractionlimit also achieved by STED-FCS will be discussed in the next section.

One of the most recent applications of the FCS diffusion law by Imaging FCS hinted at the possible role played by line tension at the boundary of domains and domain melting in the determination of dynamic membrane organization with respect to cholesterol content and temperature in SLBs respectively. ${ }^{[75]}$ Interestingly, the measurements were able to show percolation of the lipid probes in the matrix of small mobile domains which mimic meshwork compartmentalization upon increase 
of cholesterol content in an otherwise gelfluid phase separated SLB (Fig. 3a).

\subsection{Cell Membranes}

Upon establishing the FCS diffusion law framework with respect to confocal FCS, Lenne et al. applied it to characterize the diffusion behaviors of certain groups of membrane lipids and proteins (Fig. 4) and study the roles of lipids and the actin cytoskeleton in the organization of the cell plasma membrane. ${ }^{[76]}$ They revealed lesterol and sphingomyelin are two significant factors, as proposed in the 'lipid raft model', responsible for the transient confinements of sphingolipid analogs and GPI-anchored proteins in isolated microdomains. They also showed that such confinement in isolated microdomains is unaffected by meshwork compartmentalization of the actin cytoskeleton after disrupting the actin cytoskeleton. An interesting observation made on disrupting the actin cytoskeleton was the more obvious contribution of lipid-dependent microdomains in the confinement of certain transmembrane by drug and enzyme treatments that cho-

proteins such as DPP ${ }_{\text {IV. }}$ In a biologically relevant application of the confocal FCS diffusion law, Cahuzac et al. depicted the partitioning of Fas ligand, a member of the tumor necrosis factor (TNF) protein family involved in the induction of cell death upon interaction with its Fas receptor, into rafts in COS-7 cells. ${ }^{[77]}$ Increased domain partitioning of Fas was observed upon ligandreceptor interactions between Fas and its receptor. Upon depletion of cholesterol, raft partitioning of Fas was significantly diminished, possibly due to the disruption of rafts, which led to decreased induction of cell death by Fas. This indicates a possible pathway for the potency of cell death induction of Fas through its recruitment into membrane rafts to maximize its contact with Fas receptor. Another biological application of confocal FCS diffusion law exhibited the importance of the confinement of stress activating receptors into nanodomains in the plasma membrane of natural killer (NK) cells for cell tolerance. ${ }^{[78]}$

The first implementation of z-scan FCS diffusion law on live cell membrane was illustrated by probing DiD lipophilic

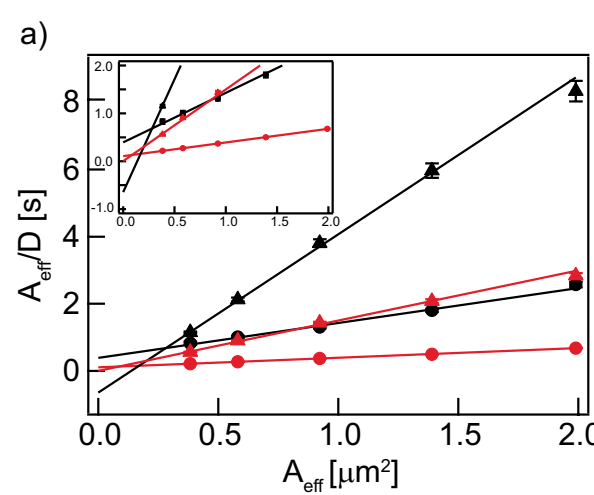

b)

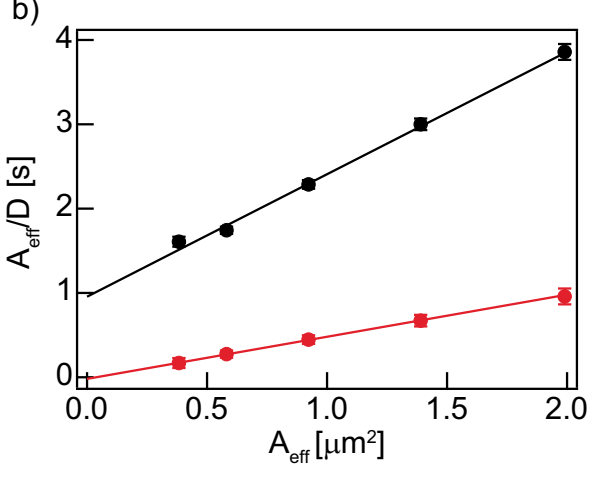

Fig. 3. Imaging FCS diffusion law plots of Rhodamine PE labeled supported lipid bilayers (a) DOPC:DPPC (1:1) (circles) and DOPC:DPPC:Cholesterol (1:1:1) (triangles) at different temperatures (black: $298 \mathrm{~K}$ and red: $313 \mathrm{~K}$ ) (Reproduced from ref. [75] with permission from Elsevier) and (b) Raft, GFP-GPI (black circles) and non-raft Dil-C ${ }_{18}$ (red circles) markers on live SH-SY5Y plasma membranes at $313 \mathrm{~K}$.
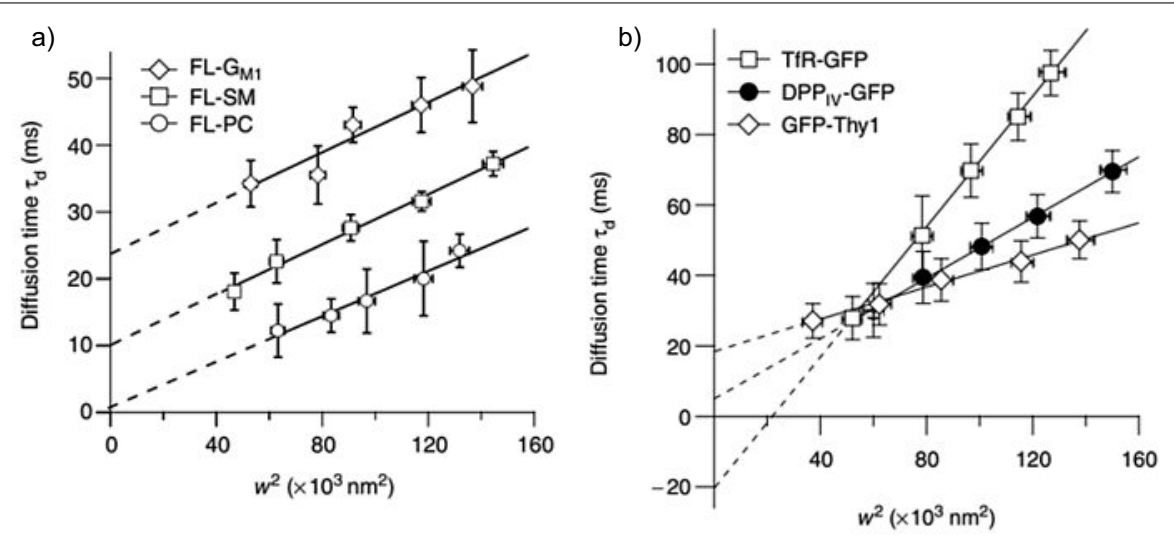

Fig. 4. Confocal FCS diffusion law plots of different lipid analogs: (a) BODIPY-C ${ }_{5}-$ phosphatidylcholine (FL-PC), BODIPY- $\mathrm{C}_{5}$-sphingomyelin (FL-SM) and BODIPY- $\mathrm{C}_{5}$-ganglioside$\mathrm{G}_{\mathrm{M} 1}\left(\mathrm{FL}-\mathrm{G}_{\mathrm{M} 1}\right)$; and membrane proteins: (b) GPI-anchored protein (GFP-Thy1) and transmembrane proteins (TfR-GFP and DPP ${ }_{I V}-$ GFP) in live COS-7 cells (Reproduced from ref. [76] with permission from John Wiley and Sons). probes in live OLN-93 cells which shows hindered diffusion by microdomain confinement. ${ }^{[57]}$ A biological application of z-scan FCS diffusion law by Ganguly and Chattopadhyay revealed the organization of serotonin $_{1 \mathrm{~A}}$ receptor on the plasma membrane of $\mathrm{CHO}-\mathrm{K} 1$ cells to be mediated by meshwork compartmentalization, indicating a possible interaction between the receptor and the actin cytoskeleton. ${ }^{[59]}$ They also showed possible structural changes of the actin cytoskeleton from the reduction of receptor compartmentalization observed upon depletion of cholesterol.

We have recently applied the Imaging FCS diffusion law to study the lateral organization of living HeLa cell membranes as a function of temperature. ${ }^{[75]}$ We observed a temperature-dependent reduction of membrane heterogeneity, perhaps due to raft melting. However, there was still a significant amount of rafts present at physiological temperature as depicted by a strong positive FCS diffusion law intercept value of a raft-localized protein. In another application, we showed that monomeric human Islet Amyloid Polypeptide (hIAPP) induces diffusion restricted domains on the plasma membrane of SH-SY5Y cells. ${ }^{[79]}$ In Fig. 3b, we show representative Imaging diffusion law plots for SH-SY5Y cells transfected with raft marker GFP-GPI and stained with non-raft lipophilic marker DiI-C $_{18}$. The Imaging FCS applications mentioned so far are performed in a TIRF setup. Recently, we were able to integrate the FCS diffusion law also in SPIM-FCS (Ng et al., unpublished data).

A breakthrough of the application of FCS diffusion law was realized by Eggeling et al. using STED-FCS where the transition regime previously not observed in any modes of diffraction-limited FCS diffusion law plots above was directly observed in live Ptk2 cell membranes. ${ }^{[66]}$ The non-linear transition of the FCS diffusion law plot for microdomain confined sphingomyelin could be captured at small detection areas of $<80 \mathrm{~nm}$ achievable by STED nanoscopy. In addition, they were also able to determine the confinement times of SM and GPI-anchored proteins within microdomains $(<20 \mathrm{~nm}$ in diameter) from the FCS diffusion law plots to be $~ 10-20 \mathrm{~ms}$. The same group, using scanning STEDFCS diffusion law, recently found membrane hotspots which transiently confine sphingolipids for a few milliseconds. ${ }^{[80]}$ Although the physiological origin of these confinement zones remains to be explored, the authors interestingly pointed out that lipid phase separation does not lead to the formation of such confinement zones. 


\section{Conclusion}

In this review, we present a brief overview of the FCS diffusion law as a membrane organization ruler. While it is mainly adapted in confocal FCS, recently it is gaining popularity in other FCS modalities which include z-scan FCS, Imaging FCS and STED-FCS. These novel variants of FCS could successfully provide insights regarding domain size, confinement time, line tension at the domain boundary and specific localization of signaling proteins and lipids. New extensions of the FCS diffusion law such as the iMSD vs. timedelay plots ${ }^{[65]}$ provide promising avenues for membrane structure elucidation. With the advancement of technology, the application regime of FCS diffusion law can be extended to decode the involvement of membrane organization in complex biological processes in the future.

\section{Acknowledgement}

X.W.N is supported by NUS graduate research scholarship. N.B is supported by a Ministry of Education (MOE) T3 grant MOE2012-T3-1-008. T.W thanks MOE for a MOE2012-T2-1-101 grant.

Received: January 10, 2015

[1] P. Mueller, D. O. Rudin, H. I. Tien, W. C. Wescott, Nature 1962, 194, 979.

[2] L. D. Frye, M. Edidin, J. Cell Sci. 1970, 7, 319

[3] S. J. Singer, G. L. Nicolson, Science 1972, 175, 720.

[4] M. J. Karnovsky, A. M. Kleinfeld, R. L. Hoover, R. D. Klausner, J. Cell Biol. 1982, 94, 1.

[5] D. A. Brown, J. K. Rose, Cell 1992, 68, 533.

[6] D. P. Cerneus, E. Ueffing, G. Posthuma, G. J. Strousz, A. van der Ende, J. Biol. Chem. 1993 268,3150

[7] R. Schroeder, E. London, D. Brown, Proc. Nat. Acad. Sci. USA 1994, 91, 12130

[8] E. Kubler, H. G. Dohlman, M. P. Lisanti, J. Biol. Chem. 1996, 271, 32975..

[9] K. Simons, E. Ikonen, Nature 1997, 387, 569.

[10] D. A. Brown, E. London, Annu. Rev. Cell Dev. Biol. 1998, 14, 111.

[11] K. Simons, D. Toomre, Nature Rev. Mol. Cell Biol. 2000, $1,31$.

[12] R. G. Parton, A. A. Richards, Traffic 2003, 4, 724.

[13] L. Rajendran, K. Simons, J. Cell Sci. 2005, 118, 1099.

[14] L. J. Pike, J. Lipid Res. 2006, 47, 1597.

[15] G. M. Lee, F. Zhang, A. Ishihara, C. L. McNeil, K. A. Jacobson, J. Cell Biol. 1993, 120, 25.

[16] S. Ladha, A. R. Mackie, L. J. Harvey, D C. Clark, E. J. A. Lea, M. Brullemans, H. Duclohier, Biophys. J. 1996, 71, 1364.

[17] A. Sonnleitner, G. J. Schütz, T. Schmidt, Biophys. J. 1999, 77, 2638.

[18] T. Fujiwara, K. Ritchie, H. Murakoshi, K. Jacobson, A. Kusumi, J. Cell Biol. 2002, 157, 1071.

[19] M. Ge, A. Gidwani, H. Brown, D. Holowka, B. Baird, J. H. Freed, Biophys. J. 2003, 85, 1278.
[20] M. J. Swamy, L. Ciani, M. Ge, A. K. Smith, D. Holowka, B. Baird, J. H. Freed, Biophys. J. 2006, $90,4452$.

[21] P. Singh, P. K. Tarafdar, M. J. Swamy, A. Chattopadhyay, J. Phys. Chem. B 2012, 116, 2999.

[22] S. W. Hui, D. F. Parsons, Science 1975, 190, 383.

[23] I. A. Prior, C. Muncke, R. G. Parton, J. F. Hancock, J. Cell Biol. 2003, 160, 165.

[24] Y. Sugimoto, H. Saito, R. Tabeta, M. Kodama, J. Biochem. 1986, 100, 867 .

[25] A. Lopez, M. P. Rols, J. Teissie, Biochem. 1988, $27,1222$.

[26] M. Auger, Curr. Issues Molecul. Biol. 2000, 2, 119.

[27] M. Wallgren, L. Beranova, Q. D. Pham, K. Linh, M. Lidman, J. Procek, K. Cyprych, P. K. J. Kinnunen, M. Hof, G. Gröbner, Faraday Discuss. 2013, 161, 499.

[28] J. Seelig, A. Seelig, Quart. Rev. Biophys. 2009, $13,19$.

[29] M. Maekawa, G. D. Fairn, J. Cell Sci. 2014 127,1

[30] T. Ueno, T. Nagano, Nature Meth. 2011, 8, 642.

[31] L. Salomé, J.-L. Cazeils, A. Lopez, J.-F. Tocanne, Eur. Biophys. J. 1998, 27, 391

[32] E. Yechiel, M. Edidin, J. Cell Biol. 1987, 105, 755.

[33] M. Edidin, I. Stroynowski, J. Cell Biol. 1991, $112,1143$.

[34] E. Sezgin, P. Schwille, Cold Spring Harbor Perspec. Biol. 2011, 3, a009803.

[35] J. Davoust, P. F. Devaux, L. Leger, EMBO J. 1982, 1,1233 .

[36] M. P. Clausen, B. C. Lagerholm, Nano Lett. 2013, 13, 2332

[37] P. J. Bosch, J. S. Kanger, V. Subramaniam, Biophys. J. 2014, 107, 588

[38] K. Murase, T. Fujiwara, Y. Umemura, K. Suzuki, R. Iino, H. Yamashita, M. Saito, H. Murakoshi, K. Ritchie, A. Kusumi, Biophys. J. 2004, 86,4075 .

[39] G. J. Schütz, G. Kada, V. P. Pastushenko, H. Schindler, EMBO J. 2000, 19, 892.

[40] M. Edidin, M. C. Zuniga, M. P. Sheetz, Proc. Nat. Acad. Sci. USA 1994, 91, 3378

[41] Y. Sako, A. Kusumi, J. Cell Biol. 1995, 129, 1559.

[42] M. J. Saxton, K. Jacobson, Ann. Rev. Biophys. Biomol. Struct. 1997, 26, 373.

[43] G M. Lee, B. Johnstone, K. Jacobson, B. Caterson, J. Cell Biol. 1993, 123, 1899.

[44] L. Wawrezinieck, H. Rigneault, D. Marguet, P.F. Lenne, Biophys. J. 2005, 89, 4029.

[45] D. Magde, E. L. Elson, W. W. Webb, Phys. Rev. Lett. 1972, 29, 705 .

[46] E. L. Elson, D. Magde, Biopolymers 1974, 13, 1.

[47] E. L. Elson, W. W. Webb, Biopolymers 1974, $13,29$.

[48] E. Klotzsch, G. J. Schütz, Phil. Trans. R. Soc. B 2013, 368, 20120033.

[49] B.-A. Truong-Quang, P.-F. Lenne, Front. Plant Sci. 2014, $5,1$.

[50] R. Macháň, T. Wohland, FEBS Lett. 2014, 588, 3571.

[51] L. Wawrezinieck, P.-F. Lenne, D. Marguet, H. Rigneault, Proc. SPIE 2004, 5462, 92.

[52] J. He, S.-M. Guo, M. Bathe, Anal. Chem. 2012 , $84,3871$.

[53] S.-M. Guo, J. He, N. Monnier, G. Sun, T. Wohland, M. Bathe, Anal. Chem. 2012, 84 , 3880.

[54] S.-M. Guo, N. Bag, A. Mishra, T. Wohland, M. Bathe, Biophys. J. 2014, 106, 190.
[55] N. Destainville, Soft Matter 2008, 4, 1288.

[56] A. Masuda, K. Ushida, T. Okamoto, Biophys. J. 2005, $88,3584$.

[57] J. Humpolíčková, E. Gielen, A. Benda, V. Fagulova, J. Vercammen, M. Vandeven, M. Hof, M. Ameloot, Y. Engelborghs, Biophys. J. 2006, 91, L23.

[58] A. Benda, M. Beneš, V. Marecek, A. Lhotsky, W. T. Hermens, M. Hof, Langmuir 2003, 9, 4120.

[59] S. Ganguly, A. Chattopadhyay, Biophys. J. 2010, 99, 1397

[60] N. Bag, T. Wohland, Ann. Rev. Phys. Chem. 2014, 65, 225

[61] A. P. Singh, T. Wohland, Curr. Opin. Chem. Biol. 2014, 20, 29

[62] B. Kannan, L. Guo, T. Sudhaharan, S. Ahmed, I. Maruyama, T. Wohland, Anal. Chem. 2007, $79,4463$.

[63] A. P. Singh, J. W. Krieger, J. Buchholz, E. Charbon, J. Langowski, T. Wohland, Optics Expr. 2013, 21, 8652

[64] N. Bag, J. Sankaran, A. Paul, R. S. Kraut, T. Wohland, ChemPhysChem 2012, 13, 2784.

[65] C. Di Rienzo, E. Gratton, F. Beltram, F. Cardarelli, Proc. Nat. Acad. Sci. USA 2013, 110, 12307.

[66] C. Eggeling, C. Ringemann, R. Medda, G. Schwarzmann, K. Sandhoff, S. Polyakova, V. N. Belov, B. Hein, C. von Middendorff, A. Schönle, S. W. Hell, Nature 2009, 457, 1159.

[67] F. Göttfert, C. A. Wurm, V. Mueller, S. Berning, V. C. Cordes, A. Honigmann, S. W. Hell, Biophys. J. 2013, 105, L01.

[68] F. Heinemann, S. K. Vogel, P. Schwille, Biophys. J. 2013, 104, 1465.

[69] F. Heinemann, P. Schwille, ChemPhysChem 2011, 12, 2568.

[70] C. Favard, J. Wenger, P.-F. Lenne, H. Rigneault, Biophys. J. 2011, 100, 1242.

[71] R. Macháň, M. Hof, T. Chernovets, M. N. Zhmak, T. V. Ovchinnikova, J. Sýkora, Anal. Bioanal. Chem. 2011, 399, 3547.

[72] M. Štefl, R. Šachl, J. Humpolíčková, M. Cebecauer, R. Macháň, M. Kolářová, L. B.Å. Johansson, M. Hof, Biophys. J. 2012, 102, 2104.

[73] S. M. Sterling, E. S. Allgeyer, J. Fick, I. Prudovsky, M. D. Mason, D. J. Neivandt, Langmuir 2013, 29, 7966.

[74] J. Wenger, H. Rigneault, J. Dintinger, D. Marguet, P.-F. Lenne, J. Biol. Phys. 2006, 32, SN1.

[75] N. Bag, D. H. X. Yap, T. Wohland, Biochim. Biophys. Acta 2013, 1838, 802.

[76] P.-F. Lenne, L. Wawrezinieck, F. Conchonaud, O. Wurtz, A. Boned, X.-J. Guo, H. Rigneault, H.-T. He, D. Marguet, EMBO J. 2006, 25, 3245.

[77] N. Cahuzac, W. Baum, V. Kirkin, F. Conchonaud, L. Wawrezinieck, D. Marguet, O. Janssen, M. Zörnig, A.-O. Hueber, Blood 2006, 107, 2384.

[78] S. Guia, B. N. Jaeger, S. Piatek, S. Mailfert, T. Trombik, A. Fenis, N. Chevrier, T. Walzer, Y. M. Kerdiles, D. Marguet, E. Vivier, S. Ugolini, Science Signal. 2011, 4, 1.

[79] N. Bag, A. Ali, V. S. Chauhan, T. Wohland, A. Mishra, Chem. Commun. 2013, 49, 9155.

[80] A. Honigmann, V. Mueller, H. Ta, A. Schoenle, E. Sezgin, S. W. Hell, C. Eggeling, Nature Commun. 2014, 5, 5412. 